\title{
BMJ Open Effects of parenting interventions for at- risk parents with infants: a systematic review and meta-analyses
}

\author{
Signe B Rayce, ${ }^{1}$ Ida S Rasmussen, ${ }^{1}$ Sihu K Klest, ${ }^{2}$ Joshua Patras, ${ }^{2}$ \\ Maiken Pontoppidan ${ }^{1}$
}

To cite: Rayce SB,

Rasmussen IS, Klest SK, et al. Effects of parenting interventions for at-risk parents with infants: a systematic review and meta-analyses. BMJ Open 2017;7:e015707. doi:10.1136/ bmjopen-2016-015707

- Prepublication history and additional material for this paper are available online. To view these files, please visit the journal online (http://dx.doi. org/10.1136/bmjopen-2016015707).

Received 27 December 2016 Revised 17 July 2017

Accepted 20 July 2017

CrossMark

${ }^{1}$ Department for Child and Family, VIVE - The Danish Centre of Applied Social Science, Copenhagen, Denmark

${ }^{2}$ Faculty of Health Sciences, University of Troms $\emptyset$, Arctic University of Norway, Troms $\emptyset$, Norway

Correspondence to Dr Maiken Pontoppidan; mpo@sfi.dk

\section{ABSTRACT}

Objectives Infancy is a critical stage of life, and a secure relationship with caring and responsive caregivers is crucial for healthy infant development. Early parenting interventions aim to support families in which infants are at risk of developmental harm. Our objective is to systematically review the effects of parenting interventions on child development and on parent-child relationship for at-risk families with infants aged 0-12 months. Design This is a systematic review and meta-analyses. We extracted publications from 10 databases in June 2013, January 2015 and June 2016, and supplemented with grey literature and hand search. We assessed risk of bias, calculated effect sizes and conducted meta-analyses. Inclusion criteria (1) Randomised controlled trials of structured psychosocial interventions offered to at-risk families with infants aged 0-12 months in Western Organisation for Economic Co-operation and Development (OECD) countries, (2) interventions with a minimum of three sessions and at least half of these delivered postnatally and (3) outcomes reported for child development or parent-child relationship.

Results Sixteen studies were included. Meta-analyses were conducted on seven outcomes represented in 13 studies. Parenting interventions significantly improved child behaviour ( $d=0.14 ; 95 \% \mathrm{Cl} 0.03$ to 0.26 ), parentchild relationship ( $d=0.44 ; 95 \% \mathrm{Cl} 0.09$ to 0.80 ) and maternal sensitivity ( $d=0.46$; $95 \% \mathrm{Cl} 0.26$ to 0.65 ) postintervention. There were no significant effects on cognitive development $(d=0.13 ; 95 \% \mathrm{Cl}-0.08$ to 0.41$)$, internalising behaviour $(d=0.16 ; 95 \% \mathrm{Cl}-0.03$ to 0.33 ) or externalising behaviour $(d=0.16 ; 95 \% \mathrm{Cl}-0.01$ to 0.30 ) post-intervention. At long-term follow-up we found no significant effect on child behaviour $(d=0.15 ; 95 \% \mathrm{Cl}$ -0.03 to 0.31 ).

Conclusions Interventions offered to at-risk families in the first year of the child's life appear to improve child behaviour, parent-child relationship and maternal sensitivity post-intervention, but not child cognitive development and internalising or externalising behaviour. Future studies should incorporate follow-up assessments to examine long-term effects of early interventions.

\section{INTRODUCTION}

The first year of a child's life is characterised by rapid development that forms the foundation for lifelong developmental trajectories.

\section{Strengths and limitations of this study}

- Comprehensive search strategy and screening procedure.

- Evaluation of child development and parent-child relationship outcomes.

- Meta-analyses conducted on seven outcomes.

- Few studies provide follow-up data.

Limited information on intervention implementation.

A healthy environment is crucial for infants' emotional well-being and future physical and mental health. ${ }^{12}$ Experiencing severe adversity early in life can alter a child's development and lead to toxic stress responses, impairing brain chemistry and neuronal architecture. ${ }^{3}$ For infants, severe adversity typically takes the form of caregiver neglect and physical or emotional abuse. The highest rates of child neglect and violent abuse occur for children younger than $5,{ }^{45}$ with the most severe cases, which involve injury or death, occurring predominantly to children under the age of $1 .^{6}$

Mental health problems are common in infants, but symptoms are often less intrusive and less distinctly identifiable than for older children. ${ }^{7-12}$ The Copenhagen Child Cohort 2000 study found a prevalence rate of $18 \%$ for axis I diagnoses (according to Diagnostic Classification (DC): 0-3) in children aged 18 months, with regulatory disorders and disturbances in parent child-relationships being the most frequent mental health diagnoses. ${ }^{8}$ The high prevalence in mental health diagnoses is important to note, as early onset of behavioural or emotional problems and adverse environmental factors increases the risk for negative outcomes later in life, such as substance abuse, delinquency, violence, teen pregnancy, school dropout, continued mental health problems and long-term unemployment. ${ }^{1} 2813-18$ 
Becoming a parent can be stressful and challenging, ${ }^{19-21}$ particularly for parents who have experienced trauma, abuse, poverty or other stressors. ${ }^{22}$ Early-intervention parenting programmes aim to assist parents with the challenges they experience. Most of these interventions teach caregivers specific strategies and skills that foster healthy child development with an emphasis on promoting warm and responsive caregiving. ${ }^{23}$

Existing systematic reviews of the effects of parenting interventions offered to families with young children have shown mixed results. ${ }^{14-29}$ In a review of 78 studies aimed at families with children aged $0-5$ years, Piquero $e t$ $a l^{14}$ found an average effect size $(g)$ of 0.37 for decreased antisocial behaviour and delinquency for intervention children. Based on 22 studies, Barlow et $a t^{28}$ concluded that there is tentative support for the effect of groupbased interventions on emotional and behavioural adjustment in children aged $0-3$ years. MacBeth $e t a l^{24}$ found medium effect sizes for child or parent outcomes in a review of the Mellow Parenting intervention for families with children aged $0-8$ years. Barlow et $a t^{26}$ found some evidence suggesting that parenting programmes for teenage parents may improve parent-child interaction. Barlow et $a l^{27}$ reviewed parent-infant psychotherapy for high-risk families with infants aged 0-24 months; they found that infant attachment improved, but they found no effects on other outcomes. Reviewing interventions offered to a universal group of parents of infants aged 0-1 year, Pontoppidan et $a l^{25}$ found mixed and inconclusive results for child development and parent-child relationship outcomes. Peacock $e t a l^{30}$ examined the effects of home visits for disadvantaged families with children aged 0-6 years and found improved child development outcomes when the intervention was implemented early.

The existing reviews include very few studies of interventions for at-risk parents that are initiated within the first year of the infants' life. Therefore, we do not know if early preventive parenting interventions are effective in improving child development or parent-child relationship outcomes. The aim of this review was to systematically review the effects of parenting interventions offered to at-risk families with infants aged 0-12 months. We included randomised controlled trials of parenting interventions reporting child development or parent-child relationship outcomes at postintervention or follow-up.

\section{METHODS}

\section{Search strategy}

This review was conducted according to the Preferred Reporting Items for Systematic Reviews and Meta-Analyses. We did not register a protocol. The database searches were performed in June 2013 and were updated in January 2015 and June 2016. We searched 10 international bibliographical databases: Campbell Library, Cochrane Library, CRD (Centre for Reviews and Dissemination), ERIC, PsycINFO, PubMed, Science Citation Index Expanded, Social Care Online, Social Science
Citation Index and SocINDEX. Operational definitions were determined for each database separately. The main search was made up of combinations of the following terms: infant*, neonat*, parent*, mother*, father*, child*, relation*, attach*, behavi*, psychotherap*, therap*, intervention*, train*, interaction, parenting, learning and education. The searches included Medical Subject Headings, Boolean operators and filters. Publication year was not a restriction. Furthermore, we searched for grey literature, hand-searched four journals and snowballed for relevant references.

\section{Eligibility criteria and study selection}

We screened all publications based on title and abstract. Publications that could not be excluded were screened based on the full-text version. Table 1 shows the inclusion and exclusion criteria.

We excluded studies that examined parenting interventions aimed at specific risk groups such as teen mothers; parents with severe mental health problems; or parents with children born preterm, at low birth weight or with congenital diseases. Families experiencing difficulties such as these have specific needs, and interventions aimed at these groups may be more targeted when compared with parenting interventions aimed at broader, at-risk groups of parents. Since our focus was parenting interventions aimed at at-risk parents in general, we excluded studies developed for specific risk groups.

Each publication was screened by two research assistants under close supervision by MP and SBR. Uncertainties regarding inclusion were discussed with MP and SBR. Screening was performed in Eppi-Reviewer V.4. ${ }^{31}$

\section{Data extraction and risk of bias assessment}

We developed a data extraction tool for the descriptive coding and extracted information on (1) study design, (2) sample characteristics, (3) setting, (4) intervention details, (5) outcome measures and (6) child age at postintervention and at follow-up. Information was extracted by one research assistant and subsequently checked by another reviewer. Disagreements were discussed with MP or SBR. Primary outcomes were child behaviour and the parent-child relationship. Secondary outcomes were other child development markers such as cognitive development, language/communication, psychomotor development, parent sensitivity and attachment classification. When reported, both total scores and subscale scores were extracted.

Numeric coding of outcome data was conducted by ISR and checked by MP or SBR. We resolved disagreements by consulting a third reviewer. Risk of bias was assessed separately for each relevant outcome for all studies based on a risk-of-bias model developed by Professor Barnaby Reeves and the Cochrane Non-Randomized Studies Method Group (BC Reeves, JJ Deeks, JPT Higgins and GA Wells, unpublished data, 2011). This extended model is organised and follows the same steps as the existing risk-of-bias model presented in the Cochrane Handbook, chapter 
Table 1 Inclusion and exclusion criteria

\begin{tabular}{|c|c|}
\hline Inclusion criteria & Exclusion criteria \\
\hline \multicolumn{2}{|l|}{ Population } \\
\hline $\begin{array}{l}\text { At-risk population of parents of infants } \\
0-12 \text { months old in western Organisation for } \\
\text { Economic Co-operation and Development } \\
\text { countries }\end{array}$ & $\begin{array}{l}\text { Studies including specific groups such as young mothers (mean age } \\
<20 \text { years), divorced parents, parents with mental health problems such } \\
\text { schizophrenia and abuse, and children born preterm, at low birth weight } \\
\text { or with congenital diseases }\end{array}$ \\
\hline \multicolumn{2}{|l|}{ Intervention } \\
\hline $\begin{array}{l}\text { Structured psychosocial parenting intervention } \\
\text { consisting of at least three sessions and initiated } \\
\text { either antenatal or during the child's first year of } \\
\text { life with at least half of the sessions delivered } \\
\text { postnatally }\end{array}$ & $\begin{array}{l}\text { Interventions not focusing specifically on parenting (eg, baby massage, } \\
\text { reading sessions with child or breastfeeding interventions), and } \\
\text { unstructured interventions (eg, home visits not offered in a structured } \\
\text { format) }\end{array}$ \\
\hline
\end{tabular}

\section{Control group}

No restrictions were imposed. All services or comparison interventions received or provided to the control group were allowed.

\section{Outcome \\ Child development and/or parent-child relationship outcomes}

8. ${ }^{32}$ The assessment was conducted by ISR and SBR. Any doubts were discussed with a third reviewer.

\section{Analyses}

We calculated effect sizes for all relevant outcomes for which sufficient data were provided. Effect sizes were reported using standardised mean differences (Cohen's d) with $95 \%$ CIs for continuous outcomes. Data included post-intervention and follow-up means, raw SD and sample size. Alternatively, t-values, F-tests, $\mathrm{X}^{2}$, $\mathrm{p}$ values, mean differences, eta-squared and $\beta$ coefficients were used. For dichotomous outcomes, we used ORs with $95 \%$ CIs as the effect size metric when presenting the effects of the individual studies. When used in meta-analyses, ORs were converted to $d$ using the method presented in Chinn. ${ }^{33}$ The data used to calculate ORs were number of events and sample sizes. We contacted the corresponding author for more information if a paper presented insufficient information regarding numeric outcomes. When available, we used data from adjusted analyses to calculate effect sizes. When using the adjusted mean difference, we used the unadjusted SD in order to be able to compare the effect sizes calculated from unadjusted and adjusted means, respectively. To calculate effect sizes, we used the Practical Meta-Analysis Effect Size Calculator developed by David B Wilson at George Mason University and provided by the Campbell Collaboration. ${ }^{34}$
Meta-analysis was performed when the intervention outcome and the time of assessment were comparable. If a single study provided more than one relevant measure or only subscales for a given meta-analysis, then the effect sizes of the respective measures were pooled into a combined measure.

Random effects inverse variance weighted mean effect sizes were applied and 95\% CIs were reported. Studies with larger sample sizes were therefore given more weight, all else being equal. Due to the relatively small number of studies and an assumption of between-study heterogeneity, we chose a random-effects model using the profile-likelihood estimator as suggested in Cornell. ${ }^{35}$ Variation in standardised mean difference that was attributable to heterogeneity was assessed with the $\mathrm{I}^{2}$. The estimated variance of the true effect sizes was assessed by the $\mathrm{Tau}^{2}$ statistic. When indication of high heterogeneity $\left(\mathrm{I}^{2}>75 \%\right)$ was found, sensitivity analyses were conducted, removing one study at a time in order to identify a potential source of heterogeneity. The small number of studies in the respective meta-analyses did not allow for subgroup analyses. Results were summarised for child development (behaviour, cognitive development, psychomotor development and communication/language) and parent-child relationship (relationship, sensitivity and attachment 


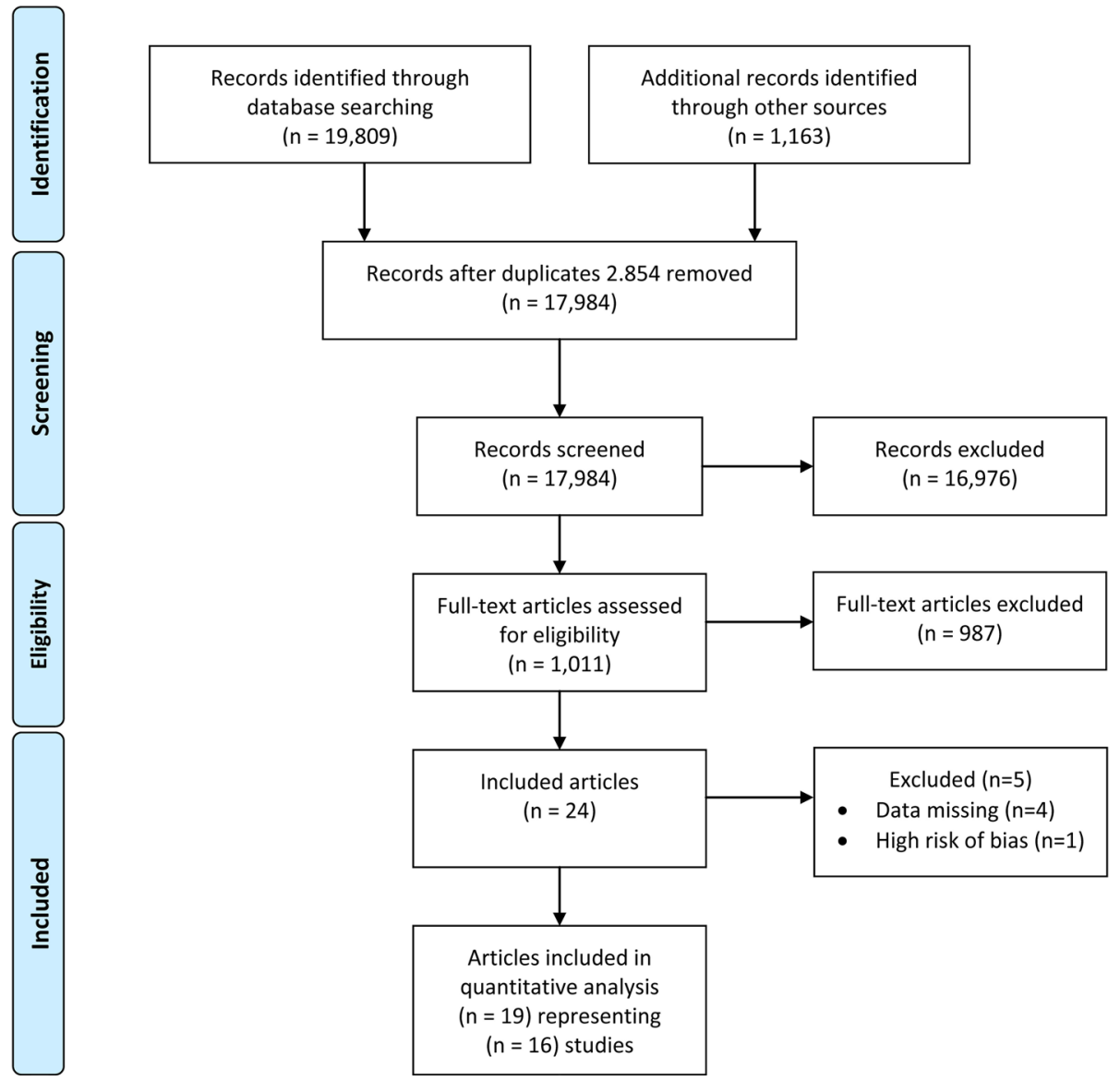

Figure 1 Flow diagram for study selection process.

classification) outcomes for the following assessment times: postintervention (PI-immediately after intervention ending), short-term (ST-less than 6 months after intervention ending), medium-term(MT-7-12 months after intervention ending) and long-term (LT-more than 12 months after intervention ending) follow-up.

\section{RESULTS}

\section{Description of studies}

The literature search identified 17984 articles after the removal of duplicates. A flow diagram for the process of study inclusion is illustrated in figure 1 . Nineteen papers representing 16 individual studies were included. ${ }^{36}$ Kaminski et $a l^{36}$ represented two trials (Los Angeles and Miami) and are handled as two studies when reporting results. Four studies were excluded, as they provided insufficient numeric data to calculate effects sizes and CIs. ${ }^{37-40}$ One study was excluded due to unacceptably high risk of bias. ${ }^{41}$

\section{Included studies}

Except for one study, ${ }^{42}$ which compared a group-based intervention with an individual-based intervention, all studies compared interventions with a no-intervention control or with treatment as usual. A few studies offered minor interventions such as psychoeducation and social worker contact to the control group. ${ }^{43-46}$ Eight studies were American, ${ }^{3642-4446-48}$ two were conducted in the Netherlands, ${ }^{49-51}$ and one study each was from Sweden, ${ }^{52-54}$ Germany, ${ }^{55}$ Italy ${ }^{56}$ New Zealand, ${ }^{57}{ }^{58}$ Norway ${ }^{45}$ and the UK. ${ }^{59}$ The oldest study was published in $1981^{47}$ and the most recent studies were published in $2015 .{ }^{4553-55}$ Sample size ranged from 40 participants ${ }^{43}$ to $755^{55}$

\section{Participant characteristics}

Table 2 shows study participant characteristics. All families exhibited at least one risk factor such as poverty, low education or living in deprived areas. Some samples were further characterised by, for example, insecure attachment, risk of developmental delay, or having a difficult or irritable infant. We did not include studies targeting families with more severe problems such as drug abuse, incarceration or chronic diseases.

Mothers' mean age ranged from 21 to 33 years. Four studies recruited primiparous mothers, ${ }^{444-5155}$ five studies also included mothers with more than one child ${ }^{4345464852-54}$ and seven studies did not report parity. ${ }^{36} 424756-59$

\section{Interventions}

Table 3 presents the intervention details. Eight studies offered individual home visits, ${ }^{44-46}$ 49-51 55-59 three studies offered individual sessions (outside the home), ${ }^{47} 48$ 52-54 one study offered group sessions, ${ }^{42}$ one study offered web coaching, ${ }^{43}$ two studies combined individual sessions and group sessions, ${ }^{36}$ and one study combined home visits 


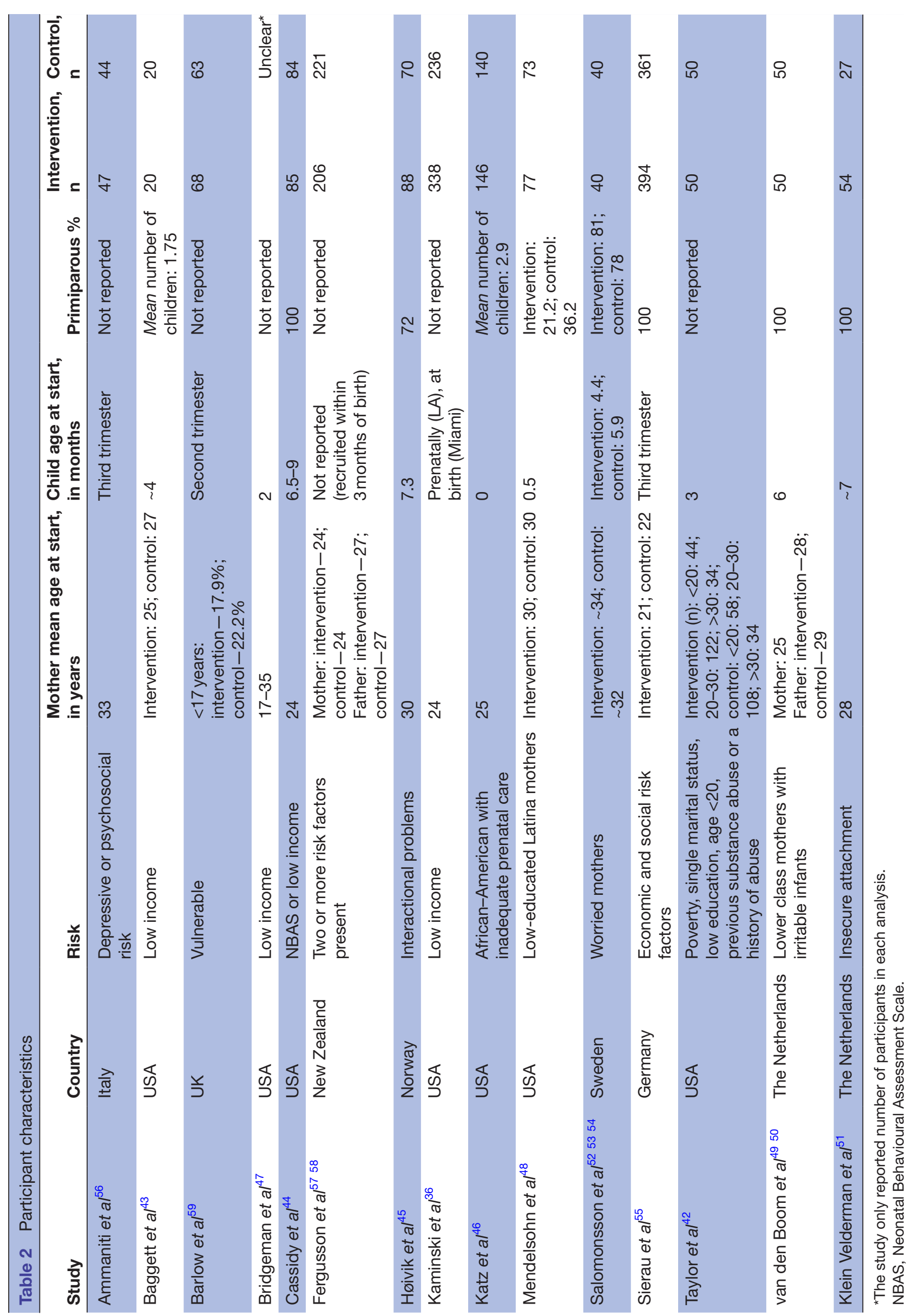




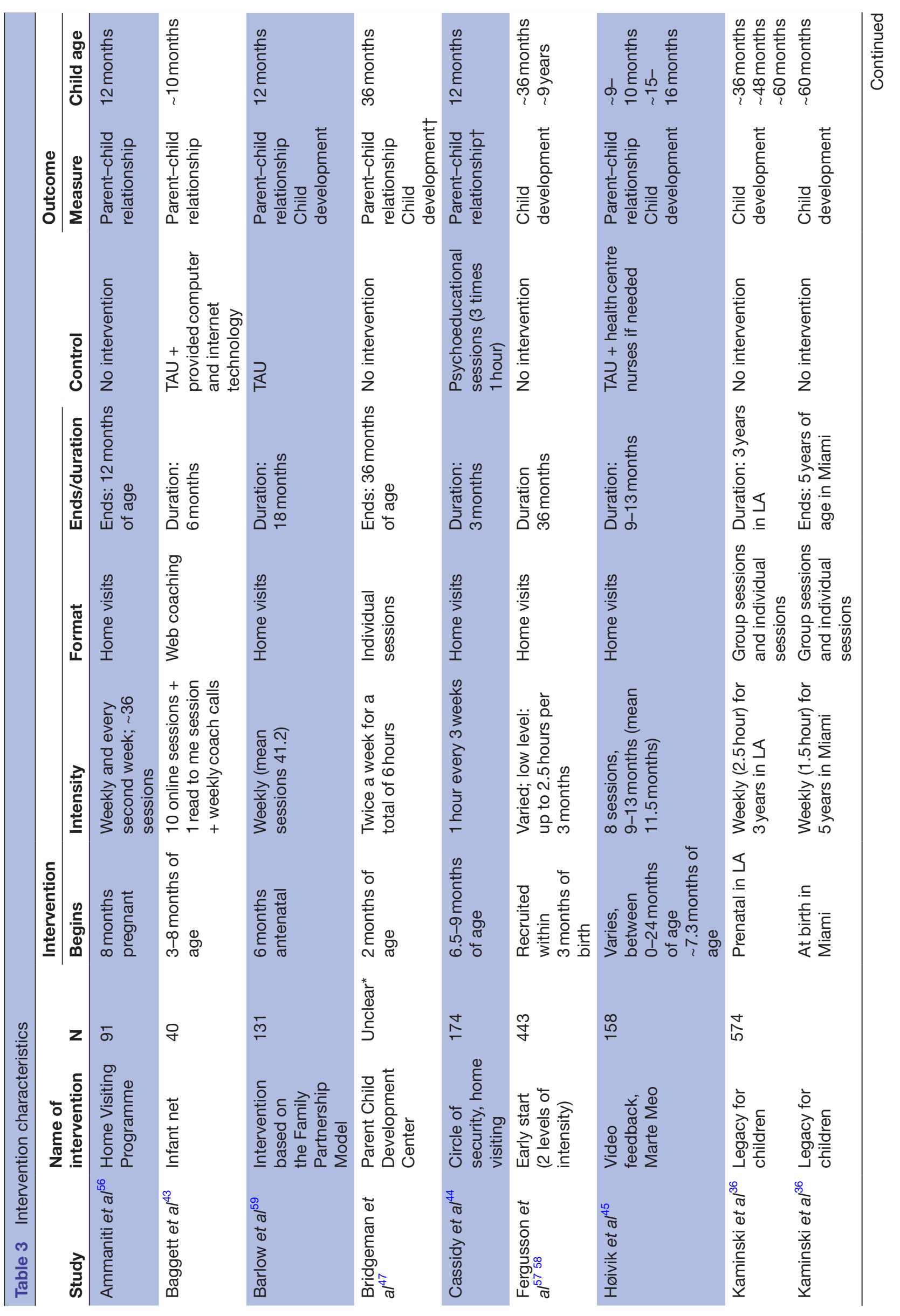




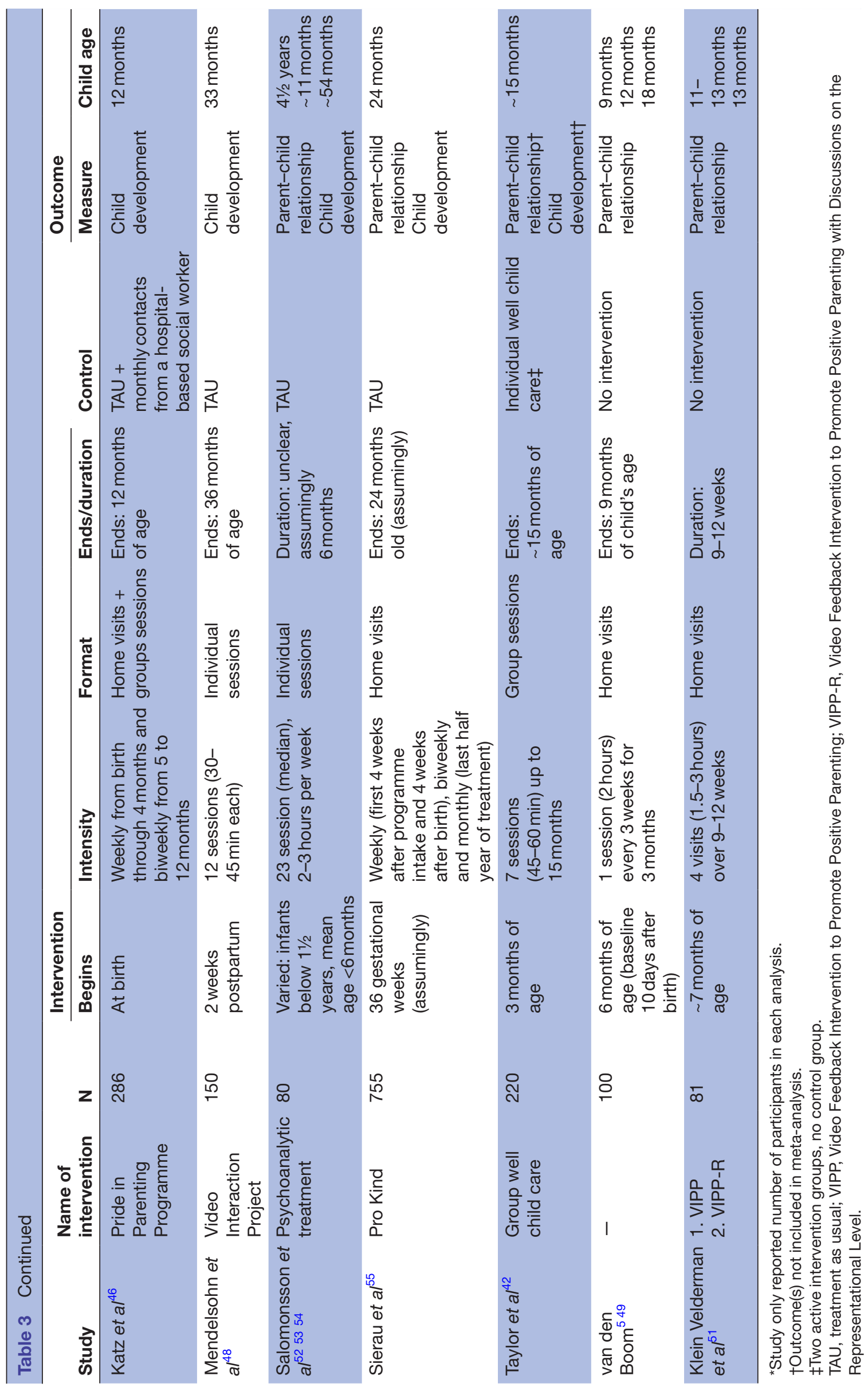


and group sessions. ${ }^{46}$ Intervention was initiated prenatally in four studies, ${ }^{36555659}$ and 12 studies initiated intervention after the child was born. ${ }^{36} 42-545758$ The duration of the interventions varied from relatively short interventions ( $\leq 6$ months) ${ }^{43} 44$ 49-54 to medium-length interventions (7-12 months) ${ }^{42} 45465659$ to long interventions ( $\geq 24$ months). ${ }^{364748555758}$

\section{Outcomes}

Child development and the parent-child relationship were measured based on parent-report questionnaires, teacher-report questionnaires, structured interviews and videos. Five studies reported only child development outcomes, ${ }^{36} 46485758$ five reported only parentchild relationship outcomes ${ }^{43} 444^{49-5156}$ and six reported both. ${ }^{42454752-5559}$ Timing of assessment was divided into four assessment times: (1) postintervention follow-up, (2) short-term follow-up, (3) medium-term follow-up and (4) long-term follow-up.

All studies reported a postintervention outcome. Two studies reported an outcome at short-term follow-up, ${ }^{45} 4950$ two at medium-term follow-up ${ }^{36} 49$ and three at long-term follow-up. ${ }^{36} 52-545758$

\section{Risk of bias}

The risk of bias assessments are shown in online supplementary table 1 and are divided into child development outcomes and parent-child relationship outcomes. Many studies provided insufficient information for at least two domains, thereby hindering a clear judgement for risk of bias. Risk of bias generally ranged between low and medium. However, three studies had outcomes where one or two domains had a moderate risk of bias. ${ }^{45-47}$ Two studies had outcomes with high risk of bias in one domain. ${ }^{45}$ Based on an overall judgement across riskof-bias domains, two outcomes (Comprehensive Test of Basic Skills Form (CTBS) math and reading scores) ${ }^{47}$ and one study ${ }^{41}$ were excluded from the review. The reasons were, on the one hand, high risk of bias in relation to 'incomplete data addressed' combined with unclear risk of bias judgements in all other domains, ${ }^{47}$ and on the other hand the pronounced baseline imbalance not being addressed. ${ }^{41}$

The outcomes included in the child development meta-analyses were characterised by low-to-medium and unclear risk-of-bias domains, whereas the meta-analyses on parent-child relationship outcomes primarily included outcomes with a relatively low or unclear risk of bias. Two studies represented in the meta-analyses of both child development and parent-child relationship outcomes had domains assessed as having moderate or high risk of bias. ${ }^{45} 47$

\section{Child development outcomes post-intervention}

Table 4 presents the study outcomes for the individual studies.

Meta-analysis of the primary outcome is reported in figure 2, and the secondary outcomes are reported in online supplementary figures.
Behaviour

The meta-analysis of parent-reported child behaviour shown in figure 2 included eight studies. ${ }^{36} 454852555859$ The analysis showed a small but significant effect on child behaviour ( $d=0.14 ; 95 \%$ CI 0.03 to 0.26$)$ favouring the intervention group. One study that offered a considerably longer intervention than the rest was removed for a sensitivity analysis, which found that the results were not substantially affected by removing the study. ${ }^{36}$ The study was therefore kept in the analysis. For the internalising and externalising subscales, no significant difference between intervention and control group was found (see online supplementary figures 2 and 3). None of the behavioural outcomes that were not included in a meta-analysis showed statistically significant differences between intervention and control group. ${ }^{46559}$

Three studies reported observer-rated child behaviour using the behavioural rating scale (BRS) from Bayley II. $^{46} 5559$ One study used a dichotomised version of BRS, ${ }^{46}$ which may not have been able to detect changes in this population since all but one (intervention) and three (control) children were rated as unproblematic. Meta-analysis was therefore not conducted. None of the studies found statistically significant effects.

\section{Cognitive development}

The meta-analysis on cognitive development included five studies (online supplementary figure 3 ). ${ }^{27} 46-4855$ There was no significant difference between intervention and control groups $(d=0.13 ; 95 \%$ CI -0.08 to 0.41$)$. A sensitivity analysis was conducted in which the one study that did not apply the Mental Developmental Index was removed, ${ }^{47}$ and the analysis found that the effect size decreased $(d=0.03)$ but remained insignificant $(95 \%$ CI -0.12 to 0.21$)$.

\section{Psychomotor development}

We could not perform meta-analysis for psychomotor development outcomes, as one study provided data comparing two active interventions. ${ }^{42}$ Of the three studies that included psychomotor development, none of them found significant effects. ${ }^{42455}$

\section{Communication/language development}

We could not perform meta-analysis for communication/ language outcomes, as the measures varied considerably. Two studies found no significant effect on communication/language development, ${ }^{48}{ }^{55}$ whereas one found significantly improved communication/language development for the intervention group $(d=0.72 ; 95 \%$ CI 0.24 to 1.20$).{ }^{47}$

\section{Child development outcomes at follow-up}

Because few studies reported child development outcomes at follow-up, we were only able to conduct a meta-analysis for one of the follow-up outcomes.

\section{Child behaviour}

The meta-analysis of parent-rated child behaviour at longterm follow-up, as shown in online supplementary figure 4, 


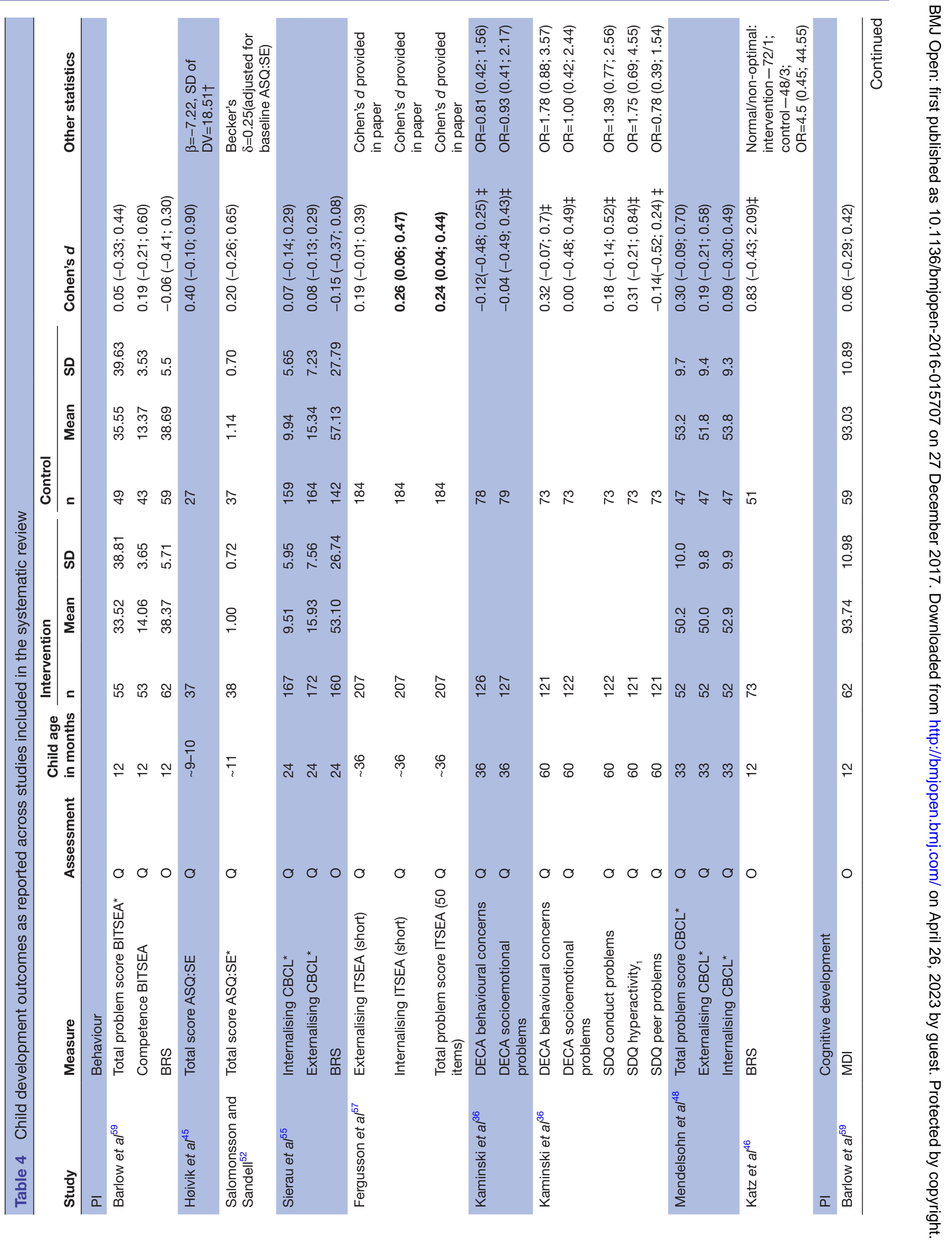




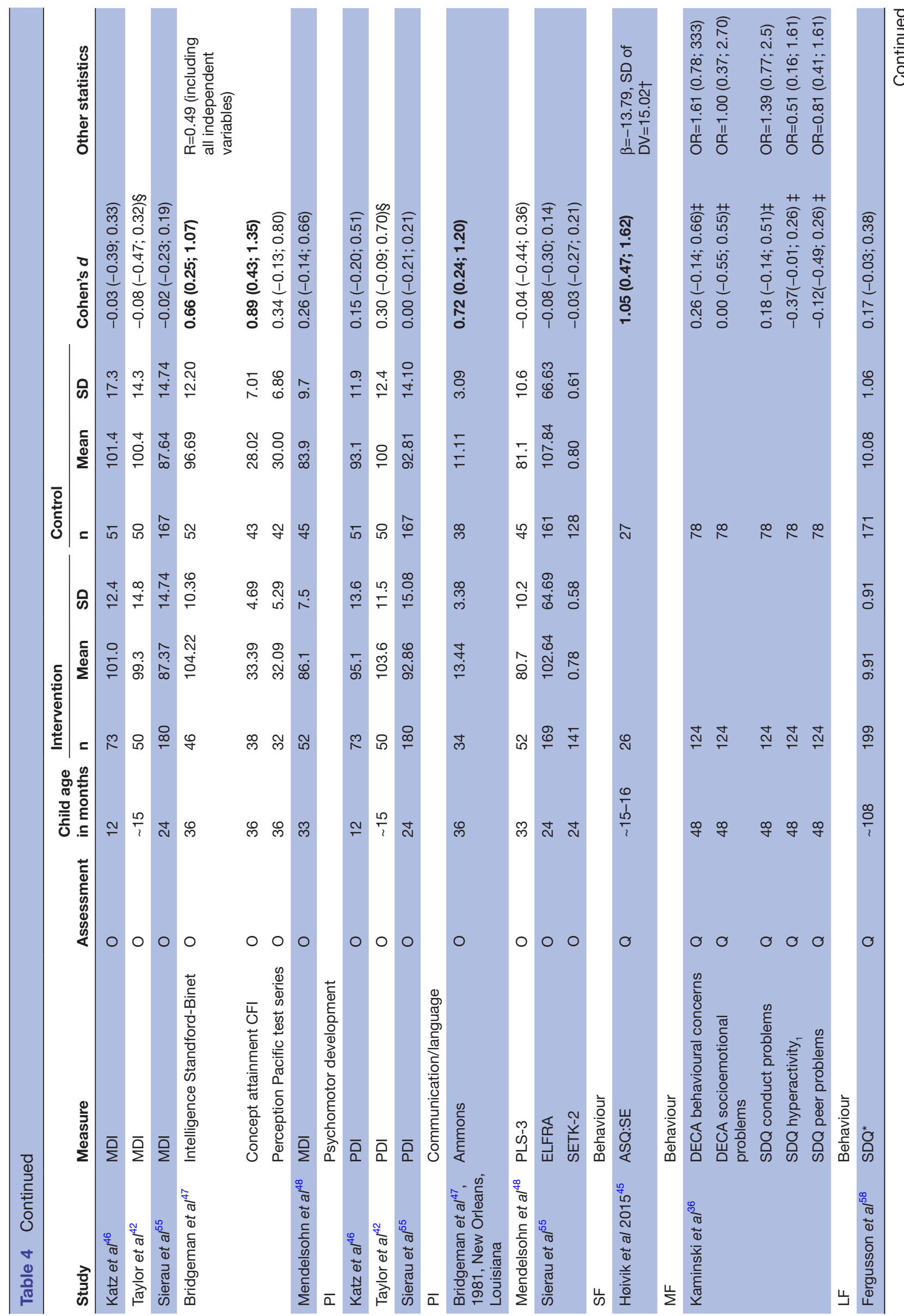




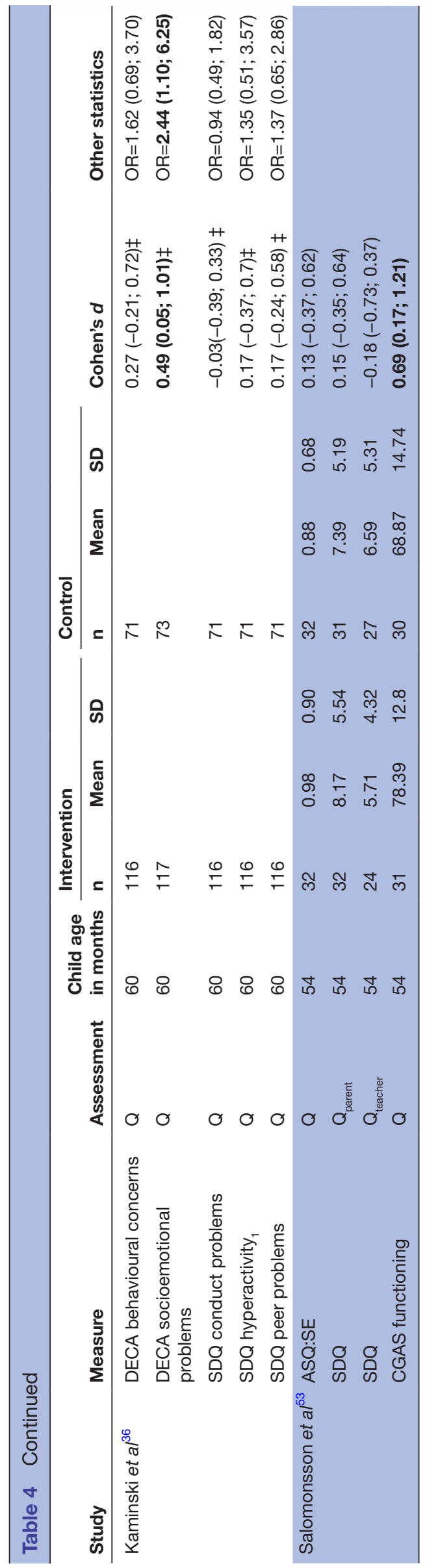

咅

要

흐음 है О

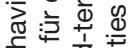

Ф

을 :ㅇ⿺ㅇ

0 $8 x^{\circ}$

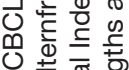

बㄴ

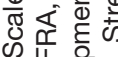

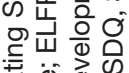

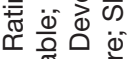

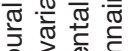

荡芒

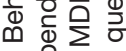

की

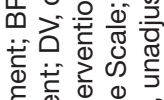

क्र

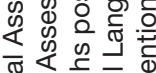

등 0

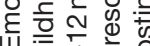

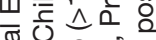

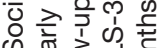

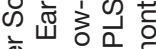

夏 $\times \overline{\overline{0}}$

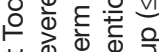

蒙守

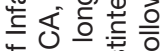

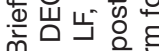

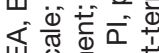
崩 可

宁产安的 을

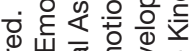
需立

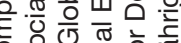

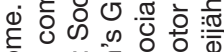

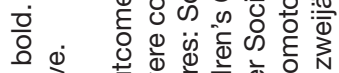

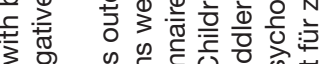

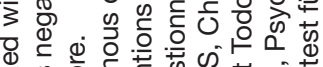

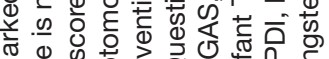
๘ँ

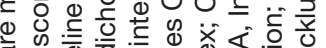

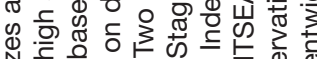
$N$ क

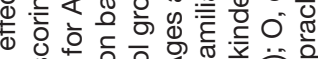
ब.

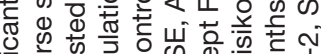

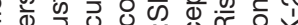

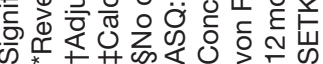




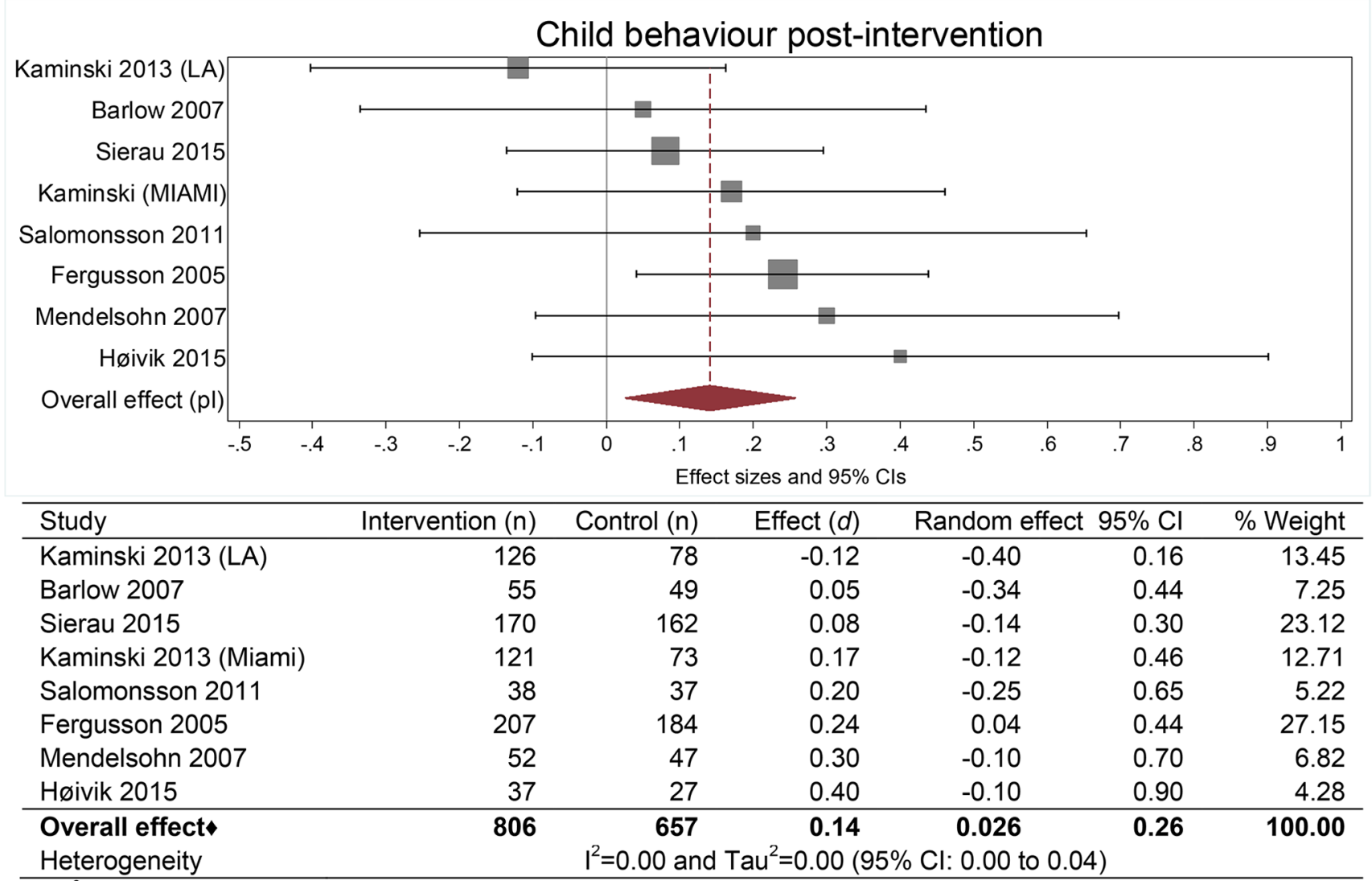

$\checkmark \mathrm{Tau}^{2}$ using Maximum likelihood was negative and set to zero. The Profile likelihood method successfully converged for CI limits.

Figure 2 Meta-analysis of studies reporting child behaviour outcomes at postintervention. ML, maximum likelihood.

included child behaviour scores (Strengths and Difficulties Questionnaires (SDQ)) from three studies. ${ }^{36535}$ No significant effect was found ( $d=0.15 ; 95 \%$ CI -0.03 to 0.31$)$.

At short-term follow-up, one study found a significant positive effect on child behaviour $(d=1.05$; $95 \%$ CI 0.47 to 1.62).$^{45}$ At medium-term follow-up, one study found no significant effects on behavioural concerns, conduct problems, hyperactivity or peer problems. ${ }^{36}$ At long-term follow-up, one study found a significant positive effect on child functioning (Children's Global Assessment Scale) $(d=0.69 ; 95 \%$ CI 0.17 to 1.21$), 5^{53}$ and one study found a significant positive effect on child socioemotional development (Devereux Early Childhood Assessment) $(\mathrm{OR}=2.44 ; 95 \%$ CI 1.10 to 6.25$){ }^{36}$

No studies reported follow-up data on cognitive development, communication/language or psychomotor development.

\section{Parent-child relationship postintervention}

Table 5 presents the study outcomes for the individual studies.

Meta-analysis of the primary outcome is reported in figure 3, and the secondary outcomes are reported in online supplementary figures.

\section{Parent-child relationship}

The meta-analysis of the overall parent-child relationship included nine studies and is presented in figure $3 .^{434547495154-5659}$ The parent-child relationship was significantly better in the intervention group as compared with the control group ( $d=0.44 ; 95 \%$ CI 0.09 to 0.80 ). The measures reported in the studies vary to some degree, which could be a source of heterogeneity. $\mathrm{I}^{2}$ was 81 , indicating that a large proportion of the observed variance in effect sizes may be attributable to heterogeneity rather than to sampling error.

\section{Maternal sensitivity}

We performed a separate meta-analysis on maternal sensitivity, which is a central component in the parent-child relationship. The meta-analysis included five studies (online supplementary figure 5) and showed a significant effect favouring the intervention group $(d=0.46 ; 95 \% \mathrm{CI}$ 0.26 to 0.65$).{ }^{4751545659}$

\section{Attachment}

Two studies reported attachment classification. ${ }^{44}$ They found no significant effects of the intervention.

\section{Parent-child relationship at follow-up}

Because few studies reported parent-child relationship outcomes at follow-up, we could not conduct meta-analyses for any parent-child relationship follow-up outcomes.

At short-term follow-up, one study found no significant effect on the parent-child relationship. ${ }^{45}$ At medium-term 


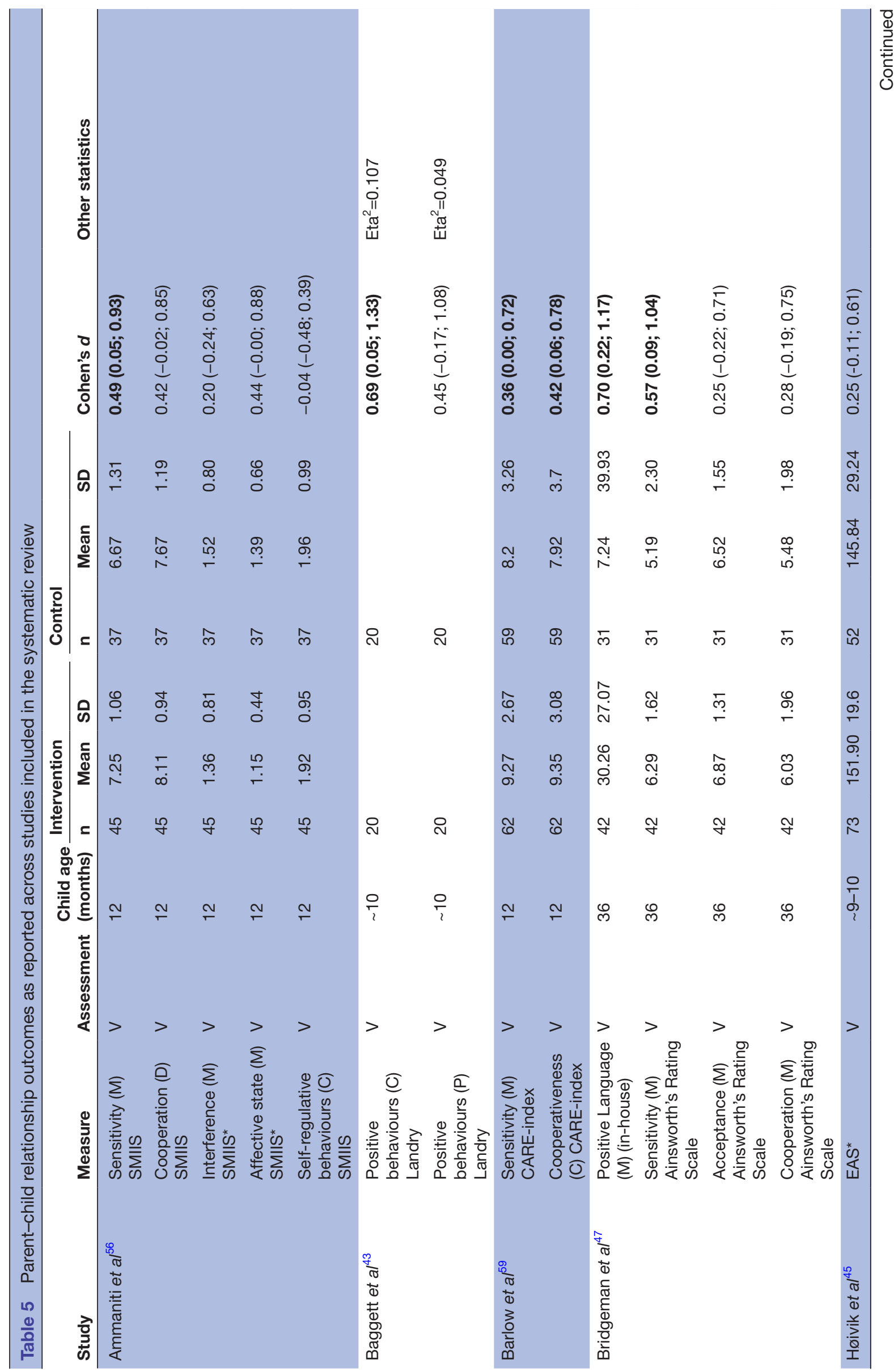




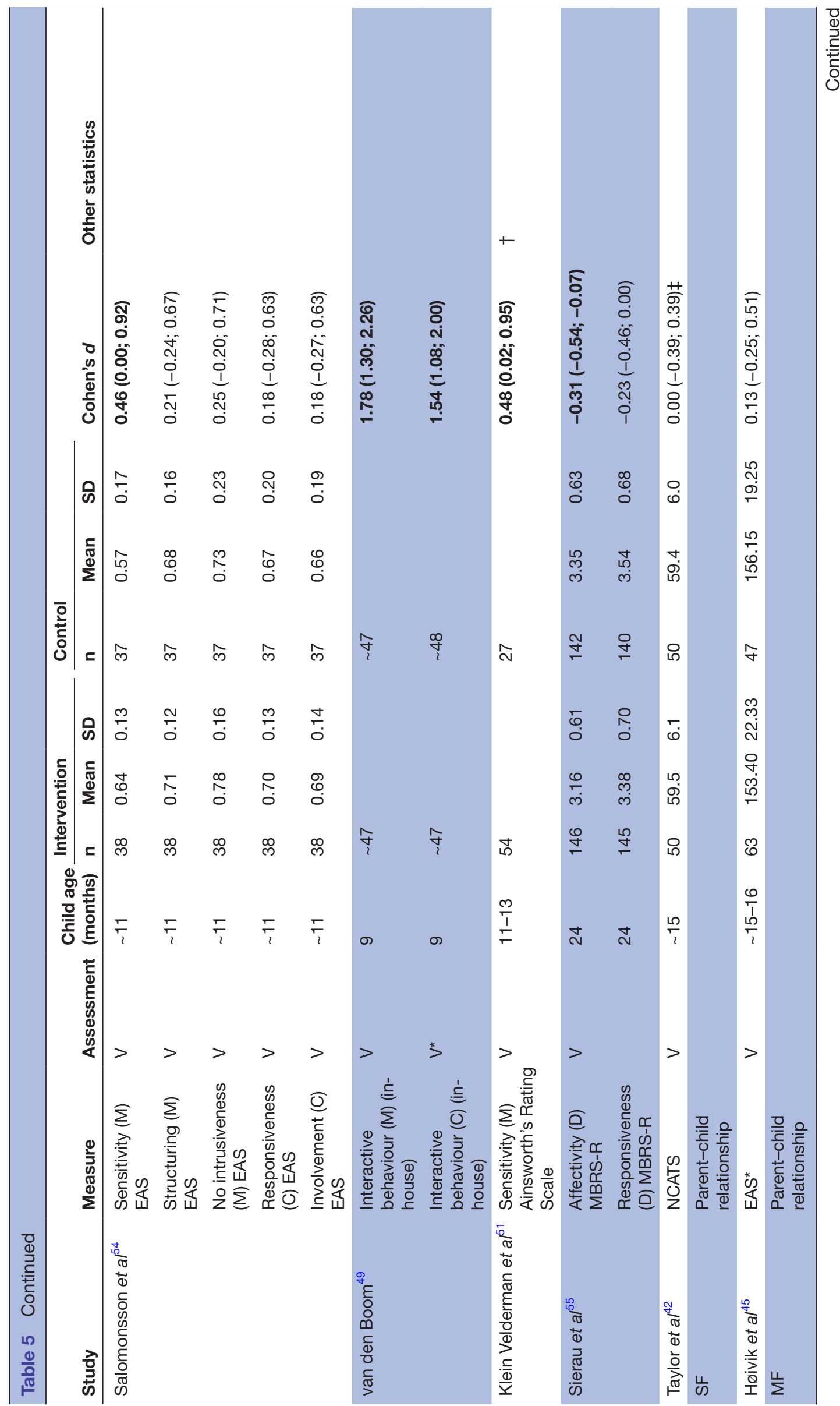

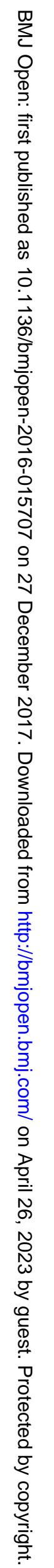




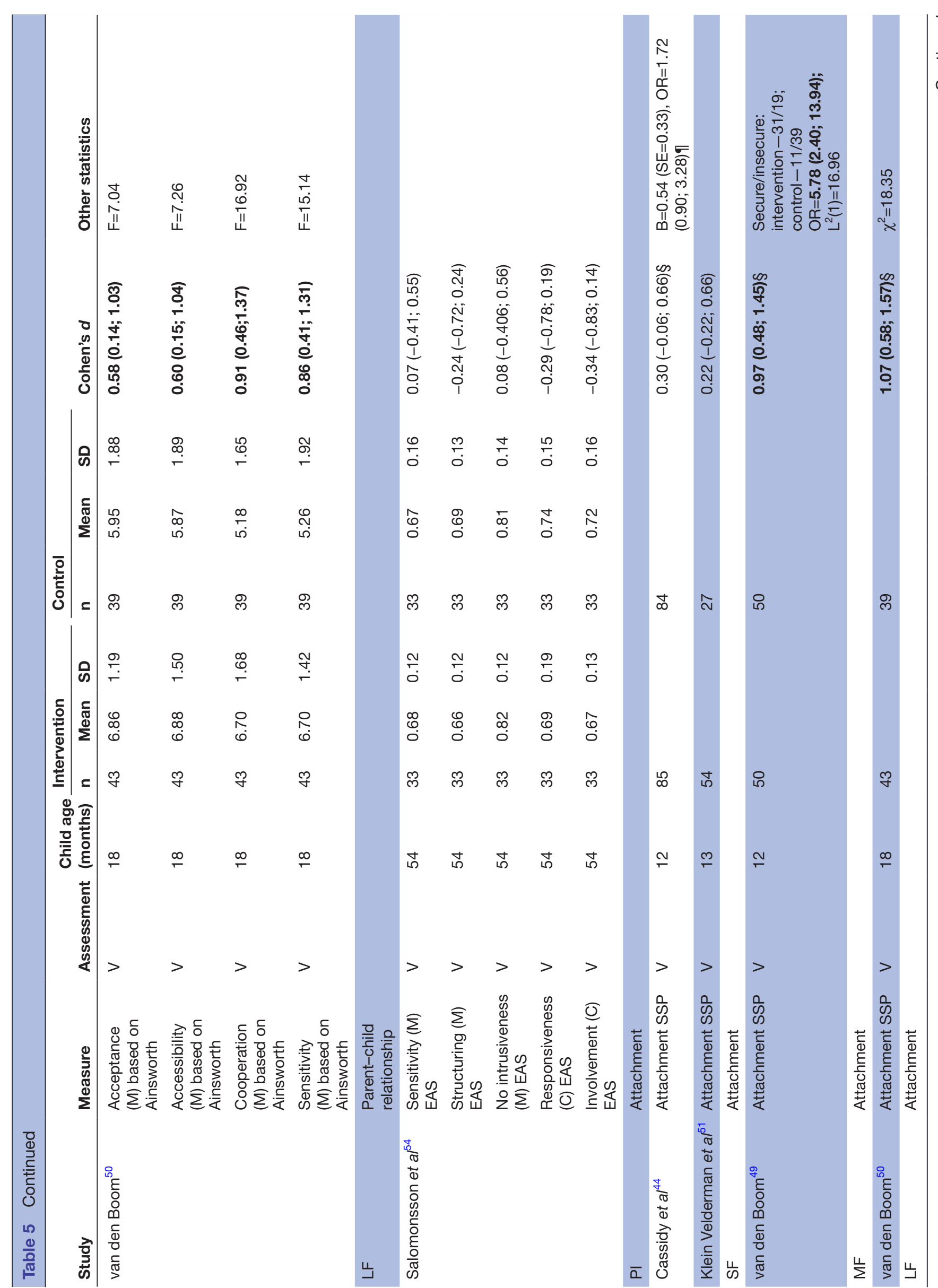




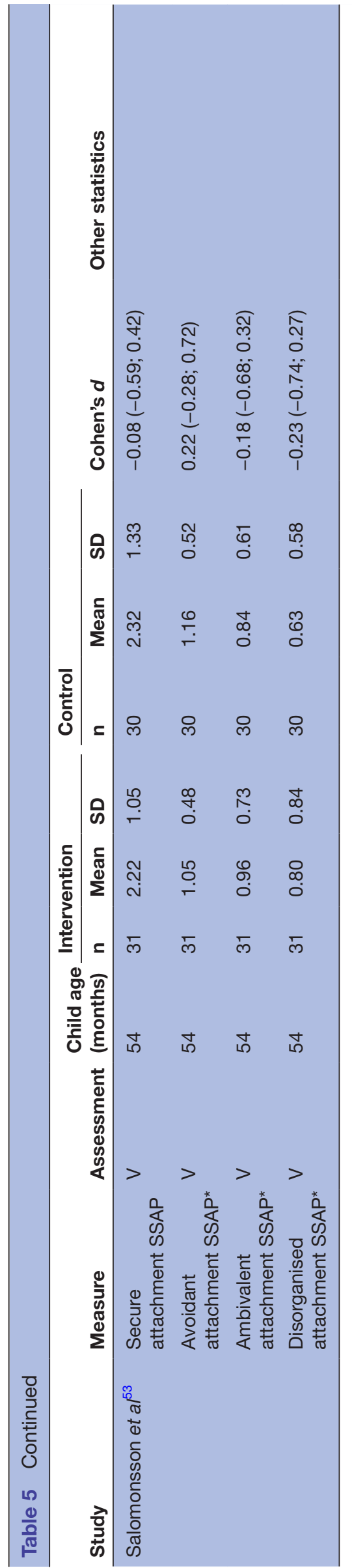

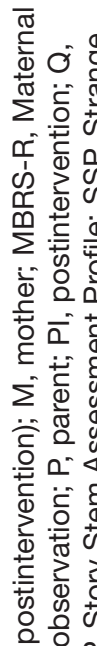

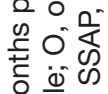

它

$\therefore$ 品

윅 $\frac{0}{0}$

วิ으응

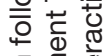

है

宁荡

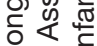

늘 을

is

$\frac{1}{\pi} \cdot \sum$

क 今ँ

का

㠃

文 $z$

읗 吾望

岌 론

का

山응

वं

른문

है

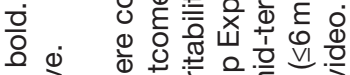

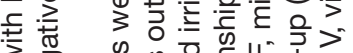

उ.

电定

ब。

ह

ब

N

क 1 造宁心

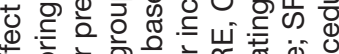

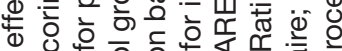

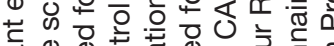

㐘

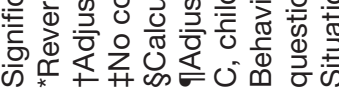




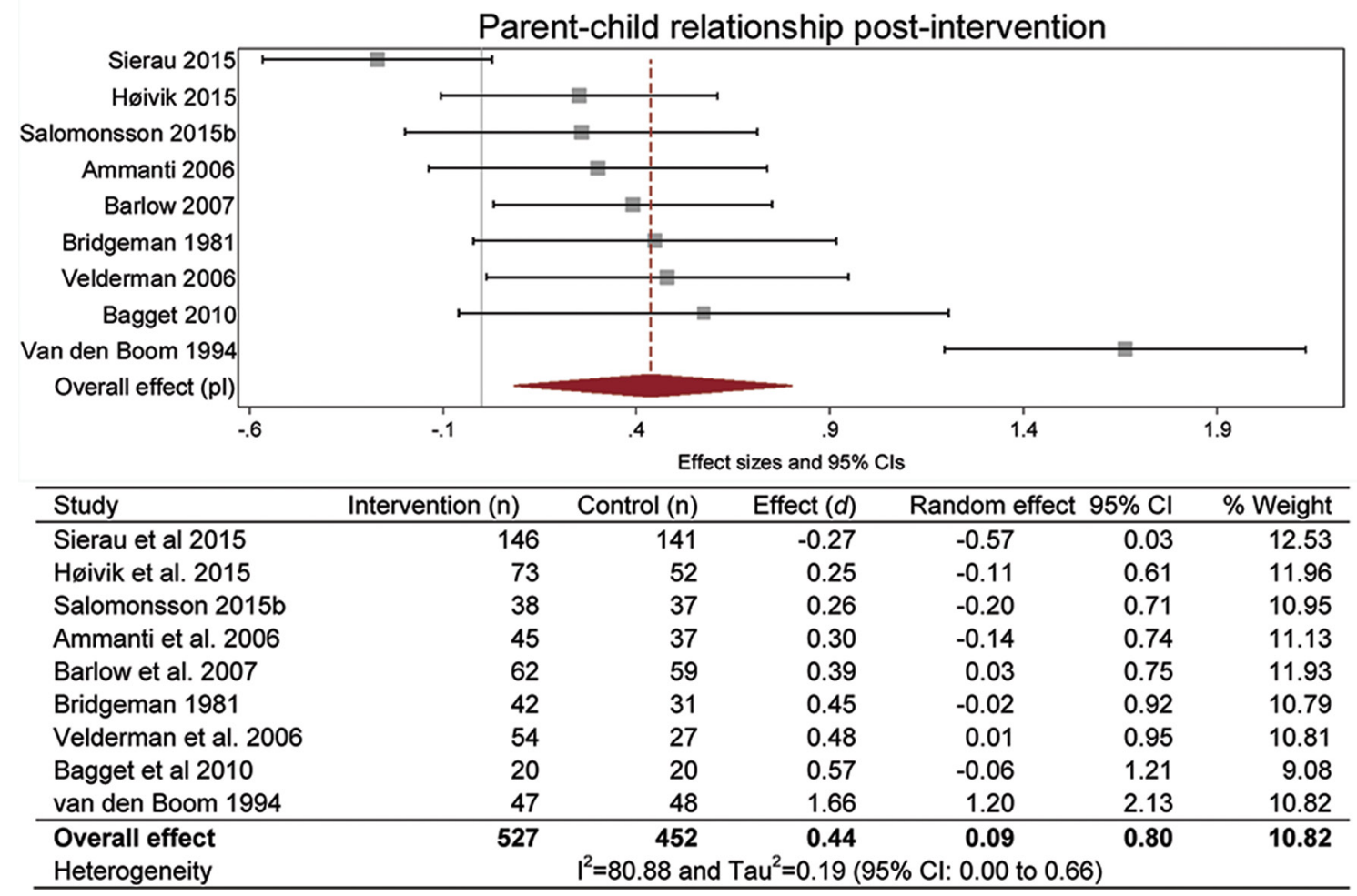

Figure 3 Meta-analysis of studies reporting parent-child relationship outcomes at postintervention.

follow-up, one study found significant positive effects on maternal acceptance $(d=0.58 ; 95 \%$ CI 0.14 to 1.03$)$, accessibility $(d=0.60 ; 95 \%$ CI 0.15 to 1.04$)$ and cooperation $(d=0.91 ; 95 \%$ CI 0.46 to 1.37$) .^{50}$ At long-term follow-up, one study did not find a significant effect on the parentchild relationship. ${ }^{54}$

\section{Maternal sensitivity}

At medium-term follow-up, one study found a significant positive effect on maternal sensitivity $(d=0.86 ; 95 \%$ CI 0.41 to 1.31$).{ }^{50}$ At long-term follow-up, one study found no significant effect on maternal sensitivity. ${ }^{54}$

\section{Attachment}

At short-term and medium-term follow-up, one study found a significant positive effect on attachment at both the 12-month follow-up ( $d=0.97 ; 95 \%$ CI 0.48 to 1.45 ) and the 18 -month follow-up ( $d=1.07 ; 95 \%$ CI 0.58 to 1.57$).{ }^{49} 50$ At long-term follow-up, one study did not find a significant effect on attachment. ${ }^{53}$

\section{Sensitivity analyses}

The meta-analysis on the parent-child relationship indicated that substantial heterogeneity may be present. Sensitivity analyses showed that one study in particular contributed to the high $\mathrm{I}^{2}$ value. ${ }^{49}$ When this study was removed from the analysis, $\mathrm{I}^{2}$ decreased from 81 to 46 . $\mathrm{Tau}^{2}$ decreased from $0.19(95 \% \mathrm{CI} 0.00$ to 0.66$)$ to 0.04 (95\% CI 0.00 to 0.19$)$. The effect size decreased to 0.26 (95\% CI 0.05 to 0.50$)$.

Two of the studies included in the meta-analyses had outcomes with domains at moderate to high risk of bias. ${ }^{45}$ Removing Bridgeman et al from the meta-analysis on child behaviour did not alter the results considerably $(d=0.12 ; 95 \%$ CI 0.01 to 0.25$)$. When removed from the analysis on cognitive development, the effect decreased but remained insignificant ( $d=0.03 ; 95 \% \mathrm{CI}-0.03$ to 0.21$)$. For the parent-child relationship the effect was almost unchanged when Bridgeman et al and Høivik et al were removed, but the CI widened ( $d=0.47 ; 95 \%$ CI 0.00 to $0.95)$. The effect on maternal sensitivity $(d=0.44 ; 95 \%$ CI 0.22 to 0.65 ) was not altered considerably by removing Bridgeman et al .

\section{Relative effects}

One study compared two active interventions: group and individual. ${ }^{42}$ The authors found no difference between the two interventions on cognitive development, psychomotor development or the parent-child relationship.

\section{DISCUSSION}

We identified 19 papers representing 16 trials that investigated the effects of parenting interventions delivered to at-risk parents of infants aged $0-12$ months. Due to the variety of outcome measures applied, not all of the 16 included studies were included in the meta-analyses. At postintervention, we found a small but significant positive effect on overall child behaviour, but no significant effects on child cognitive behaviour or the child behaviour subscales internalising or externalising. We found a medium-sized effect on overall parent-child relationship and maternal sensitivity. Most of the findings from studies that 
were not represented in the meta-analyses were not statistically significant.

The meta-analyses showed the most pronounced effect sizes for parent-child interaction and maternal sensitivity, whereas the effects on child behaviour and cognitive development were either small or not significant; however, small effect sizes can have meaningful impact on population-level outcomes. ${ }^{60}$ The non-significant outcomes for internalising and externalising behaviours were also small, but may be clinically relevant for large, at-risk populations. Most interventions provided direct support for how to improve maternal sensitivity and the relationship between parent and child (eg, Circle of Security $^{61}$ and Video feedback Intervention to promote Positive Parenting ${ }^{62}$ ). Therefore, it seems reasonable that the parent-child relationship and maternal sensitivity can be improved within a relatively short time period, whereas the effects of the interventions on child development may take longer to emerge. ${ }^{63}$

The tests for the child behaviour subscales internalising and externalising narrowly included the 0 value within the $95 \%$ CIs $(-0.03$ to 0.33 and 0.00 to 0.30 , respectively). These values suggest that similar studies to those in this review would likely produce small but positive effects. Because these analyses are based on three studies, there is a certain degree of uncertainty regarding the CIs reported. A larger sample of studies may be necessary to conclusively determine the significance of these results.

Two studies represented in the meta-analyses were assessed as having a moderate to high risk of bias in one ${ }^{47}$ or two ${ }^{45}$ domains. As this could potentially affect the credibility of the results, we conducted sensitivity analyses to investigate these studies' contribution to the effect sizes. However, removing these studies from the analyses did not substantially alter the effects.

The outcomes applied in the individual studies vary and most meta-analyses are based on heterogeneous measures. Although the measures vary, they do measure the same underlying construct and can therefore be meaningfully combined in the meta-analyses.

The meta-analyses of parent-child relationship and maternal sensitivity included in-house measures, that is, measures developed by the evaluators that have, to our knowledge, not been formally validated. This could potentially affect the results; however, sensitivity analyses showed that removing these outcomes from the analyses did not substantially alter the results, therefore we kept the outcomes in the analyses.

The number of studies in the meta-analyses ranged from three to nine. While a meta-analysis on nine studies is fairly reliable, a meta-analysis including only three studies may provide a less accurate estimate of the overall effect. ${ }^{64}$ We therefore applied the random-effects model using the profile-likelihood estimator. This has been recommended for meta-analyses with a small number of studies, because it generates wider CIs than the frequently applied DerSimonian-Laird estimator. ${ }^{35}$ The results of the meta-analyses including fewer studies should still be interpreted with some caution.

This review focuses on interventions for adult mothers; studies with young mothers were excluded, including central studies such as the Olds $e t a l^{63}$ studies of Nurse Family Partnership. Although teen mothers are an at-risk group due to their age, and they often face additional risk factors such as poverty, low education and single parenthood, we have not included them in this review. We believe this is the appropriate method because teen mothers are a distinct group requiring targeted care that is developmentally appropriate for their stage in life. We consider the narrower focus on adult mothers to be a strength, because the interventions aimed at adult mothers most often differ considerably from interventions for teen mothers; this specificity reduces heterogeneity in study outcomes that are often present between the teen and adult interventions.

The included studies were conducted in countries with different levels of service for families with infants; therefore, it may not be possible to reproduce effects in other contexts. The interventions examined in the studies also varied according to approach, intensity and duration. Both short and extensive interventions were included in all meta-analyses, and we found no apparent tendencies in the results. Due to the relatively low number of studies in the meta-analyses, we could not conduct subgroup analyses. Subgroup analyses are important as they provide information about whether the effect of an intervention is modified by certain circumstances or characteristics of the participants. Eight of the included studies reported some kind of subgroup or moderator analyses. ${ }^{4-495156}$

Most of the studies did not address implementation in their design. This presents challenges with regard to assessing outcomes, as results may have been moderated, both positively and negatively, by implementation quality. Of the 16 studies reviewed, four provided information about efforts to support implementation, such as strategies to reduce participant attrition, ${ }^{46}$ information about variability in the number of intervention sessions that some families received, ${ }^{43} 4655$ and information on the intervention. ${ }^{495055}$ All of the studies could have included more information about the implementation context and the possible moderating factors associated with different strategies. Without more extensive implementation information, replicability remains problematic, particularly in circumstances where implementation supports were not well documented.

A further limitation of the study is that although many studies reported outcomes during the intervention period and postintervention, only a few reported follow-up data. We were able to perform meta-analysis for one long-term outcome: child behaviour measured by the SDQ. The analysis included three studies and found no significant difference between intervention and control groups. Individual study results at different follow-up times were mixed and therefore inconclusive for both child development and the parent-child relationship at long-term follow-up. 
It is problematic that the studies did not assess long-term outcomes because it makes it impossible to evaluate the short-term, medium-term and long-term effects of the interventions. Conclusions based on postintervention assessments may be insufficient to draw firm conclusions about the effectiveness of parenting interventions.

\section{CONCLUSION}

This review identified 16 studies that evaluated the effects of parenting interventions for at-risk caregivers with infants aged 0-12 months on child development and parent-child relationship. Meta-analyses revealed a small but statistically significant positive effect of the interventions on child behaviour as well as moderate effects on the parent-child relationship and maternal sensitivity. There were no statistically significant effects on child cognitive development, internalising behaviour or externalising behaviour at post-intervention; however, internalising and externalising behaviours were marginally significant and may have reached statistical significance with a larger sample. Similarly, the effect on child behaviour at long-term follow-up was not significant, but approaching statistical significance. Parenting interventions initiated in the child's first year of life appear to have the potential to improve child behaviour and the parent-child relationship post-intervention.

Few studies assessed child development and parentchild relationship outcomes at follow-up; therefore, it remains unclear whether parenting interventions delivered in this population will have lasting effects. Future studies should incorporate follow-up assessments to examine the long-term effects of early interventions for at-risk families.

Acknowledgements The authors would like to acknowledge and thank information specialist Anne-Marie Klint Jørgensen and Bjørn Christian Viinholt Nielsen for running the database searches, Rikke Eline Wendt for being involved in the review process, Therese Lucia Friis, Line Møller Pedersen and Louise Scheel Hjorth Thomsen for conducting the screening, and senior researcher Trine Filges and researcher Jens Dietrichson for statistical advice.

Contributors SBR co-led the review process, contributed to study design, screening, data extraction and data synthesis, performed risk of bias judgement and meta-analysis, drafted the first manuscript, critically revised the manuscript, and approved the final manuscript as submitted. ISR contributed to study design, contributed to screening, data extraction and data synthesis, performed risk of bias, drafted the first manuscript, critically revised the manuscript, and approved the final manuscript as submitted. SKK and JP contributed to study design and data synthesis, critically revised the manuscript, and approved the final manuscript as submitted. MP conceptualized and designed the study, co-led the review process, contributed to screening, data extraction and data synthesis, drafted the first manuscript, critically revised the manuscript, and approved the final manuscript as submitted.

Funding SBR and ISR were supported by a grant from the Danish Ministry of Social Affairs and the Interior. MP was supported by the Danish Ministry of Social Affairs and the Interior and grant number 7-12-0195 from TrygFonden.

Competing interests None declared.

Provenance and peer review Not commissioned; externally peer reviewed.

Data sharing statement № additional data are available.

Open Access This is an Open Access article distributed in accordance with the Creative Commons Attribution Non Commercial (CC BY-NC 4.0) license, which permits others to distribute, remix, adapt, build upon this work non-commercially, and license their derivative works on different terms, provided the original work is properly cited and the use is non-commercial. See: http://creativecommons.org/ licenses/by-nc/4.0/

(C) Article author(s) (or their employer(s) unless otherwise stated in the text of the article) 2017. All rights reserved. No commercial use is permitted unless otherwise expressly granted.

\section{REFERENCES}

1. ZeanahCH, Zeanah P. The scope of infant mental health. Zeanah $\mathrm{HJ}$, ed. Handbook of infant mental health. New York: The Guilford Press, 2009.

2. Center on the Developing Child at Harvard University. The Foundations of Lifelong Health Are Built in Early Childhood, 2010.

3. Center on the Developing Child at Harvard. Building core capabilities for life: The science behind the skills adults need to succeed in parenting and in the workplace. $2016 \mathrm{http}: / / \mathrm{www}$.developingchild. harvard.edu.

4. Corby B. Child abuse: towards a knowledge base. Philadelphia: Open University Press, 2000.

5. Geffner R, Igelman RS, Zellner J. The effects of intimate partner violence on children. New York: Haworth Maltreatment \& Trauma Press, 2003.

6. Grøgaard J. Dype Spor. Norway: Oslo: Barne og familiedepartementet, 2007.

7. Carter AS, Briggs-Gowan MJ, Davis NO. Assessment of young children's social-emotional development and psychopathology: recent advances and recommendations for practice. J Child Psychol Psychiatry 2004;45:109-34

8. Skovgaard AM, Houmann T, Christiansen E, et al. The prevalence of mental health problems in children 1(1/2) years of age - the Copenhagen Child Cohort 2000. J Child Psychol Psychiatry 2007;48:62-70.

9. Briggs-Gowan MJ, Carter AS, Bosson-Heenan J, et al. Are infanttoddler social-emotional and behavioral problems transient? J Am Acad Child Adolesc Psychiatry 2006;45:849-58.

10. Wakschlag LS, Danis B. Characterizing early childhood disruptive behavior. Zeanah $\mathrm{CH}$, ed. Handbook of infant mental health. New York, NY US: Guilford Press, 2009:392.

11. National Scientific Council on the Developing Child. 2012. Establishing a Level Foundation for Life: Mental Health Begins in Early Childhood. Working paper No: 6.

12. Bolten MI. Infant psychiatric disorders. Eur Child Adolesc Psychiatry 2013;22:69-74.

13. Skovgaard AM, Olsen EM, Christiansen E, et al. Predictors (0-10 months) of psychopathology at age 11/2 years - a general population study in The Copenhagen Child Cohort CCC 2000. J Child Psychol Psychiatry 2008;49:553-62.

14. Piquero AR, Jennings WG, Diamond $B$, et al. A meta-analysis update on the effects of early family/parent training programs on antisocial behavior and delinquency. J Exp Criminol 2016;12:229-48.

15. Conti G, Heckman JJ, et al. Economics of child well-being. In: Ben-Arieh A, Casas F, Frønes I, eds. Handbook of child well-being. Dordrecht, Netherlands: Springer, 2014:363-401.

16. Heckman JJ. The case for investing in disadvantaged young children. In: Darling-Hammond L, Grunewald R, Heckman JJ, eds. et al. Big ideas for children: investing in our nation's future. Washington, DC: First Focus, 2008:49-58.

17. Dishion TJ, Shaw D, Connell A, et al. The family check-up with highrisk indigent families: preventing problem behavior by increasing parents' positive behavior support in early childhood. Child Dev 2008;79:1395-414

18. Barlow J, Parsons J. Group-based parent-training programmes for improving emotional and behavioural adjustment in 0-3 year old children. Cochrane Database Syst Rev 2003:CD003680.

19. Coleman PK, Karraker KH. Self-efficacy and parenting quality: findings and future applications. Dev Rev 1998;18:47-85.

20. Cowan CP, Cowan PA. Interventions to ease the transition to parenthood. Why they are needed and what they can do. Fam Relat 1995;44:412-23.

21. Petch J, Halford WK. Psycho-education to enhance couples' transition to parenthood. Clin Psychol Rev 2008;28:1125-37.

22. University $\mathrm{C}$ on the DCAH. Building core capabilities for life: The science behind the skills adults need to succeed in parenting and in the workplace. 2016.

23. Kazdin AE, Weisz JR. Introduction. Context, Background, and Goals. Evidence-based psychotherapies for children and adolescents. New York: Guilford Press, 2010:3-9. 
24. MacBeth A, Law J, McGowan I, et al. Mellow Parenting: systematic review and meta-analysis of an intervention to promote sensitive parenting. Dev Med Child Neurol 2015;57:1119-28.

25. Pontoppidan M, Klest SK, Patras J, et al. Effects of universally offered parenting interventions for parents with infants: a systematic review. BMJ Open 2016;6:e011706.

26. Barlow J, Smailagic N, Bennett C, et al. Individual and group based parenting programmes for improving psychosocial outcomes for teenage parents and their children. Cochrane Database Syst Rev 2011(3):CD002964.

27. Barlow J, Bennett C, Midgley N, et al. Parent-infant psychotherapy for improving parental and infant mental health. Cochrane Database Syst Rev 2015;1:CD010534.

28. Barlow J, Bergman H, Kornør H, et al. Group-based parent training programmes for improving emotional and behavioural adjustment in young children. Cochrane Database Syst Rev 2016;(8):CD003680.

29. Kersten-Alvarez LE, Hosman $\mathrm{CMH}$, Riksen-Walraven JM, et al. Which preventive interventions effectively enhance depressed mothers' sensitivity? A meta-analysis. Infant Ment Health J 2011;32:362-76.

30. Peacock S, Konrad S, Watson E, et al. Effectiveness of home visiting programs on child outcomes: a systematic review. BMC Public Health 2013;13:17.

31. Thomas J, Brunton J, Graziosi S. EPPI-Reviewer 4: software for research synthesis. London: EPPI-Centre Software. http://eppi.ioe. ac.uk/cms/Default.aspx?alias=eppi.ioe.ac.uk/cms/er4 2010.

32. Green $\mathrm{S}$, Higgins $\mathrm{P}$, Alderson $\mathrm{P}$, et al. Cochrane handbook: cochrane reviews: Ch 8: assessing risk of bias in included studies. In: Cochrane handbook for: systematic reviews of interventions, 2011:3-10.

33. Chinn S. A simple method for converting an odds ratio to effect size for use in meta-analysis. Stat Med 2000;19:3127-31.

34. Wilson DB. Practical Meta-Analysis Effect Size Calculator. https:// www.campbellcollaboration.org/escalc/html/EffectSizeCalculator-H ome.php.

35. Cornell JE. Random-effects meta-analysis of inconsistent effects. In response. Ann Intern Med 2014;161:380.

36. Kaminski JW, Perou R, Visser SN, et al. Behavioral and socioemotional outcomes through age 5 years of the legacy for children public health approach to improving developmental outcomes among children born into poverty. Am J Public Health 2013;103:1058-66

37. Wagner MM, Clayton SL. The parents as teachers program: results from two demonstrations. Futur Child 1999:9:91-115.

38. Wasik BH, Ramey CT, Bryant DM, et al. A longitudinal study of two early intervention strategies: project CARE. Child Dev 1990;61:1682-96.

39. Casey PH, Whitt JK. Effect of the pediatrician on the mother-infant relationship. Pediatrics 1980;65:715-20.

40. Roggman LA, Boyce LK, Cook GA. Keeping kids on track: impacts of a parenting-focused early head start program on attachment security and cognitive development. Early Educ Dev 2009;20:920-41.

41. Infante-Rivard C, Filion G, Baumgarten M, et al. A Public health home intervention among families of low socioeconomic status. Children's Health Care 1989:18:102-7.

42. Taylor JA, Davis RL, Kemper KJ. A randomized controlled trial of group versus individual well child care for high-risk children: maternal-child interaction and developmental outcomes. Pediatrics 1997;99:e9-6.

43. Baggett KM, Davis B, Feil EG, et al. Technologies for expanding the reach of evidence-based interventions: Preliminary results for promoting social-emotional development in early childhood. Topics Early Child Spec Educ 2010;29:226-38.

44. Cassidy J, Woodhouse SS, Sherman LJ, et al. Enhancing infant attachment security: an examination of treatment efficacy and differential susceptibility. Dev Psychopathol 2011;23:131-48.

45. Høivik MS, Lydersen S, Drugli MB, et al. Video feedback compared to treatment as usual in families with parent-child interactions problems: a randomized controlled trial. Child Adolesc Psychiatry Ment Health 2015:9:3

46. Katz KS, Jarrett MH, El-Mohandes AA, et al. Effectiveness of a combined home visiting and group intervention for low income African American mothers: the pride in parenting program. Matern Child Health J 2011;15 Suppl 1:75-84.

47. Bridgeman B, Blumenthal JB, Andres SR. Parent Child Development Center: final evaluation report. Alabama (Birmingham); Louisiana (New Orleans); Michigan (Detroit); 1981.

48. Mendelsohn AL, Valdez PT, Flynn V, et al. Use of videotaped interactions during pediatric well-child care: impact at 33 months on parenting and on child development. J Dev Behav Pediatr 2007:28:206-12.

49. van den Boom DC. The influence of temperament and mothering on attachment and exploration: an experimental manipulation of sensitive responsiveness among lower-class mothers with irritable infants. Child Dev 1994;65:1457-77.

50. van den Boom DC. Do first-year intervention effects endure? Followup during toddlerhood of a sample of Dutch irritable infants. Child Dev 1995;66:1798-816.

51. Klein Velderman M, Bakermans-Kranenburg MJ, Juffer F, et al. Effects of attachment-based interventions on maternal sensitivity and infant attachment: differential susceptibility of highly reactive infants. $J$ Fam Psychol 2006;20:266-74.

52. Salomonsson B, Sandell R. A randomized controlled trial of mother-infant psychoanalytic treatment: I. Outcomes on selfreport questionnaires and external ratings. Infant Ment Health $J$ 2011;32:207-31.

53. Salomonsson MW, Sorjonen K, Salomonsson B. A long-term followup of a randomized controlled trial of mother-infant psychoanalytic treatment: outcomes on the children. Infant Ment Health $J$ 2015;36:12-29.

54. Salomonsson MW, Sorjonen K, Salomonsson B. A long-term follow-up study of a randomized controlled trial of mother-infant psychoanalytic treatment: outcomes on mothers and interactions. Infant Ment Health J 2015;36:542-55.

55. Sierau S, Dähne V, Brand T, et al. Effects of home visitation on maternal competencies, family environment, and child development: a randomized controlled trial. Prevention Science 2016;17:40-51.

56. Ammaniti M, Speranza AM, Tambelli R, et al. A prevention and promotion intervention program in the field of mother-infant relationship. Infant Ment Health J 2006;27:70-90.

57. Fergusson DM, et al. Randomized Trial of the Early Start Program of Home Visitation. Pediatrics 2005;116:e803-e809.

58. Fergusson DM, Boden JM, Horwood LJ. Nine-Year follow-up of a home-visitation program: a randomized trial. Pediatrics 2013;131:297-303.

59. Barlow J, Davis H, Mclntosh E, et al. Role of home visiting in improving parenting and health in families at risk of abuse and neglect: results of a multicentre randomised controlled trial and economic evaluation. Arch Dis Child 2007;92:229-33.

60. Embry DD, Vaccines B. Behavioral Vaccines and evidence-based kernels: nonpharmaceutical approaches for the prevention of mental, emotional, and behavioral disorders. Psychiatr Clin North Am 2011;34:1-34.

61. Ramsauer B, Lotzin A, Mühlhan C, et al. A randomized controlled trial comparing Circle of Security Intervention and treatment as usual as interventions to increase attachment security in infants of mentally ill mothers: Study Protocol. BMC Psychiatry 2014;14:24

62. Juffer F, Bakermans-Kranenburg MJ, Van lizendoorn MH. Promoting positive parenting: An attachment-based intervention. Routledge 2012.

63. Olds DL, Sadler L, Kitzman H. Programs for parents of infants and toddlers: recent evidence from randomized trials. J Child Psychol Psychiatry 2007;48:355-91.

64. Borenstein M, Hedges LV, Higgins JPT, et al. A basic introduction to fixed-effect and random-effects models for meta-analysis. Res Synth Methods 2010;1:97-111. 